\title{
Metodologias ativas de aprendizagem: práticas no ensino da Saúde Coletiva para alunos de Medicina
}

\author{
Active learning methodologies: practices in collective health education for medicine students
}

Ada Ávila Assunção' (1) | avilaufmg@gmail.com

\begin{abstract}
RESUMO
Introdução: Combinados à cultura geracional dos alunos a conclamar novos desafios para as escolas médicas, estão as reformas do modelo assistencial e os novos padrões de adoecimento. Essas transformações justificaram movimentos nos anos 1990 para reorganizar o ensino médico. Paralelamente, inovações no campo educacional foram alimentadas, entre outros referenciais, na teoria sociocultural. A interseção educação, pedagogia e psicologia alimentou o reconhecimento de três dimensões do processo de aprendizagem humana: plano interpsíquico, ou seja, compartilhamento entre indivíduos; mediações de objetos e imagens, ou seja, a semiótica; e o plano intrapsíquico que é relativo à internalização do conhecimento construído. Os avanços teóricos e as tecnologias instrucionais impulsionaram a aplicação de metodologias ativas de aprendizagem, que têm sido contrastadas ao modelo tradicional da aula expositiva nas escolas médicas.
\end{abstract}

Relato de experiência: Este relato apresenta experiências docentes no desenvolvimento e na aplicação de metodologias ativas de aprendizagem no ensino da Saúde Coletiva para alunos de Medicina.

Discussão: Baseando-se na experiência de adequação do modelo pedagógico da Faculdade de Medicina da Universidade Federal de Minas Gerais, três métodos foram discutidos: elaboração de projetos, sala de aula invertida e instrução por colegas, bem como os seus fundamentos pedagógicos. Examinaram-se a coerência entre valores da atual geração de alunos, as mudanças de paradigma na prestação dos serviços de saúde e as práticas de inovações pedagógicas combinadas ao emprego das tecnologias da informação e comunicação (TIC) nos projetos de ensino. A sala de aula invertida e a instrução por colegas apoiadas pelas TIC conferiram um novo formato à sala de aula, de maneira a tornar a aprendizagem mais significativa. Hesitações recorrentes por parte dos alunos foram interpretadas como tensões entre a passividade em que se encontram no modelo tradicional e a chamada para maior implicação quando são utilizadas as metodologias ativas de aprendizagem. Foi requerido trabalho docente intenso para fazer a transposição didática. Deficiências das habilidades comunicacionais do professor devem ser superadas.

Conclusão: O uso de metodologias ativas de aprendizagem, além de coerente com a cultura dessa geração de alunos, contribui, de maneira significativa, para o desenvolvimento de competências centrais na formação do aluno de Medicina. Para o futuro próximo, serão imprescindíveis avaliação sistemática da proposta e mais recursos tecnológicos.

Palavras-chave: Aprendizagem; Material de Ensino; Educação Médica; Educação Superior; Saúde Coletiva.

\begin{abstract}
Introduction: The generational culture has urged new challenges for medical schools to be tackled, including reforms of the care model and addressing new patterns of illness. These changes justified movements in the 1990s to reorganize medical education. Meanwhile, innovations in education have been encouraged, among other sources of reference, in the sociocultural theory on the relationships between the learning process and higher psychological functions. The intersection of education, pedagogy and psychology has led to recognition of three dimensions of the human learning process: interpsychic plane, that is, sharing between individuals; mediations of objects and images, that is, semiotics; and the intrapsychic plane that is related to the internalization of constructed knowledge. Theoretical advances and instructional technologies have boosted teaching models based on active learning methodologies (ALM), which have been contrasted with the traditional model of lecturing in medical schools.
\end{abstract}

Experience report: To present teaching experiences in the development and application of ALM in collective health education for medical students.

Discussion: Based on the experience of adapting the pedagogical model of the Federal University of Minas Gerais School of Medicine, three methods were discussed: project design, flipped classroom and peer instruction, as well as their pedagogical foundations. We examined the coherence between the values of the current generation of students, paradigm shifts in the provision of health services and the practices of pedagogical innovations combined with the use of information and communication technologies in teaching projects. The flipped classroom and peer instruction supported by information and communication technologies gave a new format to the classroom in order to make learning more meaningful. Recurrent hesitations by students were interpreted as tensions between their passivity in the traditional model and the call for greater implication when ALMs are used. Intense teaching work was required to make the didactic transposition. Deficiencies in the teacher's communication skills must be overcome.

Conclusion: The use of ALM, besides being consistent with the culture of this generation of students, contributes significantly to the development of core competencies in the training of medical students. For the near future, systematic evaluation of the proposal and more technological resources will be necessary.

Keywords: Learning; Teaching Materials; Medical Education; Higher Education; Public Health.

1 Universidade Federal de Minas Gerais, Belo Horizonte, Minas Gerais, Brasil.

Editora-chefe: Rosiane Viana Zuza Diniz.

Editor associado: Roberto Zonato Esteves.

Recebido em 12/01/21; Aceito em 18/05/21.

Avaliado pelo processo de double blind review. 


\section{INTRODUÇÃO}

O enfrentamento de desafios na esfera da pedagogia com conhecimentos sobre a mente humana é uma das contribuições da escola de Lev Vygotsky (1896-1934), psicólogo soviético nascido na Bielorrússia'. Modelos pedagógicos têm sido elaborados em sintonia com o reconhecimento das três dimensões do processo de aprendizagem humana: plano interpsíquico, ou seja, compartilhamento entre indivíduos; mediações de objetos e imagens, ou seja, a semiótica; e plano intrapsíquico que é relativo à internalização do conhecimento construído².

Se a dimensão sociocultural está na base da construção do conhecimento, então o aprendizado não começa na sala de aula. Na prática, essa ideia indica que cada aluno assiste à exposição de um tema pelo professor de acordo com a sua maneira própria de entender.

Inovações teóricas e avanços na tecnologia instrucional motivaram críticas a modelos pedagógicos centrados no professor e na sua comunicação unilateral com o aluno. Aula expositiva, por vezes, magistral, é uma tradição no ensino superior que ainda se mantém seja pela ideia de que é a forma mais eficaz de transmitir conhecimentos complexos, seja pela crença de que seria o método mais proveitoso para a memorização pelos alunos.

A geração dos alunos de hoje nasceu depois de 1990, sendo educada em ambientes digitais. Por isso, está acostumada a captar informações de múltiplos emissores que se sobrepõem num mesmo instante ${ }^{3}$. Essa geração é capaz de interligar vários pontos quando aborda um problema, de modo a adotar um comportamento do tipo multitarefa. Comparados à geração dos seus professores, os alunos de hoje vivenciam muito desconforto quando expostos a uma única fonte de informação, quanto mais se a exposição for prolongada ${ }^{4}$. A geração nativa digital, nos termos de Doueihi ${ }^{5}$, é ágil e hábil para manejar as ferramentas digitais. Mas não somente isso. Essa geração é capaz de usar eficazmente múltiplas funções integradas, característica fundamental para entender, ao menos em parte, o desassossego com que ela enfrenta a longa e unilateral palestra de um professor. Desde os anos 1970, vale lembrar, já era conhecida a fraqueza da capacidade de concentração e de memorização do conteúdo quando o aluno assistia passivamente à exposição unilateral do professor ${ }^{6}$. Naquela época, havia evidências de diminuição da atenção dos alunos após os primeiros dez minutos de aula expositiva ${ }^{3}$.

Combinadas à cultura geracional a conclamar novos desafios para as escolas médicas, estão as demandas sociais, as mudanças demográficas e as reformas do modelo assistencial ${ }^{7}$. A implantação de um sistema universal organizado sob os princípios da equidade e da atenção integral praticada por equipes multiprofissionais de saúde demandou mudanças nos atos de saúde ${ }^{8}$. Nos processos de reforma curricular, propalou-se a necessidade de ampliar/deslocar o eixo da formação centrada no modelo médico-paciente para a integralidade da atenção. Não seria surpreendente, pois, o efeito que as inovações do modelo assistencial provocaram sobre o modelo da formação de profissionais de saúde.

De certa forma, o desafio da substituição do tradicional modelo de organização dos sistemas de saúde está correlacionado ao desafio de elaborar um sistema formativo adaptado para atender às evoluções das necessidades de saúde de uma dada sociedade? .

As diretrizes curriculares da Faculdade de Medicina da Universidade Federal de Minas Gerais (UFMG), de 2008, orientaram utilizar "metodologias ativas de aprendizagem e articulação entre o ensino, a pesquisa e a extensão/ assistência"10. Essas metodologias fazem parte de processos educacionais centrados na participação de quem aprende em contraposição ao modelo tradicional baseado na transmissão de informações ${ }^{11,12}$. As situações didáticas são produzidas para desenvolver capacidade de interpretação e intervenção sobre a realidade, além de colaboração em equipe ${ }^{13}$.

Se, no modelo tradicional, o professor domina a classe no intuito de apresentar um conteúdo que será "absorvido" pelo aluno que o observa e escuta, os modelos centrados no aluno o estimulam a investigar e interagir com o conteúdo em vez de assumir uma postura passiva na sala de aula. As estratégias de aprendizagem ativa ampliam oportunidades de engajamento dos alunos em seu próprio aprendizado ao estimularem a autorreflexão em colaboração com os colegas, o professor e outros agentes no ambiente de aprendizagem. Estimular o aluno a pensar sobre o conteúdo e sobre a atividade que está fazendo é um método para desenvolver habilidades de resolução de problemas, bem como propicia experiências de enfrentamento de incertezas. Lidar com as incertezas de forma segura é uma competência fundamental no exercício da medicina ${ }^{14}$. Vale observar que esse aprendizado está em sintonia com as atuais demandas do trabalho em equipe multiprofissional nos serviços de saúde. As metodologias ativas de ensino são adequadas ao novo cenário da formação médica.

Neste relato, apresentam-se as experiências docentes no desenvolvimento e na aplicação de metodologias ativas de aprendizagem no ensino da Saúde Coletiva para alunos de Medicina.

\section{RELATO DE EXPERIÊNCIA}

Com base na experiência de adequação e constante aperfeiçoamento do modelo pedagógico da Faculdade de Medicina da UFMG, três métodos são examinados à luz da experiência recente: elaboração de projetos, sala de 
aula invertida e instrução por colegas, bem como os seus fundamentos pedagógicos.

Além do registro da nossa experiência na condução da disciplina Saúde do Trabalhador, que integra o ensino da Saúde Coletiva na Faculdade de Medicina da UFMG, a busca na literatura atual identificou experiências similares sobre a aplicação de metodologias ativas no desenvolvimento do ensino da Saúde Coletiva no Brasil.

O texto está organizado em quatro tópicos. Depois de apresentar a relevância da teoria sociocultural para os educadores, destacam-se os atributos e a justificativa do modelo de ensino baseado nas metodologias ativas de aprendizagem. As ideias inovadoras nesse âmbito são desenvolvidas com os argumentos relacionados à conversão no modo de ver o mundo engendrada pela combinação da técnica digital com a cultura da geração do século XXI. Na segunda parte, o foco se detém nos desafios das escolas médicas diante das profundas transformações ocorridas na sociedade, com ênfase nas adequações da estrutura dos sistemas de saúde ao perfil epidemiológico. No âmbito da prática pedagógica, a terceira parte diz respeito ao emprego das metodologias ativas de aprendizagem e ao uso da sala de aula de um modo diferente daquele centrado no professor que ministra conteúdo para alunos contemplativos. Ao final, apresentam-se obstáculos percebidos, dificuldades e exigências de intenso trabalho docente requerido no desenvolvimento da nova proposta.

\section{MODELOS E TÉCNICAS EMPREGADAS}

\section{Ensino por meio de projetos focados em problemas}

Entre os objetivos da formação do aluno da Faculdade de Medicina da UFMG, consta "Reconhecer a saúde como direito e atuar de forma a garantir a integralidade da assistência entendida como conjunto articulado e contínuo de ações e serviços preventivos e curativos, individuais e coletivos, exigidos para cada caso em todos os níveis de complexidade do sistema". Qual modelo pedagógico adotar para favorecer a construção das referidas habilidades e competências?

Ainda que mantido o recurso à ministração do conteúdo pelo professor diante de uma sala de aula em que os alunos permanecem em atitude contemplativa, programas articulados pelos setores saúde e educação, como será apresentado mais adiante, induziram esforço dos professores da Saúde Coletiva em praticar metodologias ativas de aprendizagem.

Diferentes modalidades já foram experimentadas no ensino da Saúde Coletiva, seja em estágios curriculares, seja no desenvolvimento de atividades intramuros de formato misto ${ }^{15-20}$. Atividades extramuros "puras", como é o caso dos estágios, ou intercaladas com ações na sala de aula, como a elaboração de projetos, substituíram o ato puramente teórico e informativo da aula ministrada pelo professor de Saúde Coletiva.

No primeiro caso, veja-se a experiência do Programa de Educação pelo Trabalho para a Saúde (PET-Saúde) que impulsionou em várias regiões do país a integração ensinoserviço. Alunos, tutores e professores no PET-Saúde foram estimulados a intervir numa realidade em constante interação com os agentes do processo $0^{9,19,21}$. Nessa experiência formativa, as atividades articuladas ao modelo assistencial vigente fomentaram reflexões sobre a pertinência e o alcance das ações em saúde pública. A atuação direta com as equipes de saúde no território é uma situação rica para incitar o aluno a compreender a relevância do planejamento e da gestão em saúde. Saberes experimentados à medida que o aluno se depara com as necessidades da população-alvo são significativos, de forma a prepará-lo para o exercício profissional futuro.

No segundo caso, a disciplina Saúde Coletiva e correlatas usualmente adotam metodologias mistas, que intercalam visitas técnicas e ações na sala de aula, com a finalidade de gerar projetos relativos a uma realidade sanitária específica ${ }^{16}$. A Figura 1 apresenta um esquema do planejamento das ações, que são exemplificadas a seguir, no processo de ensino em situação.

A elaboração de projetos no ambiente da prestação dos serviços de saúde é uma estratégia de aprendizagem complexa, uma vez que o aluno é exposto a uma situação desconhecida, uma unidade de saúde, por exemplo, para entender o seu funcionamento ${ }^{22}$. Estuda-se a gestão locorregional consultando, por exemplo, parâmetros de cobertura populacional estimada pelas equipes de atenção básica. $A$ atividade de projeto pode se ater ao diagnóstico ou a uma etapa específica que é atribuída a um grupo de alunos, como sistematizar informações sobre doses de vacinas aplicadas e taxas de abandono de vacinação. Outro grupo de alunos pode se dedicar à produção científica sobre indicadores em saúde, como artigos sobre relação entre cobertura da atenção básica e taxa de imunização. Procedimentos de coleta sistematizada de dados foram utilizados, por exemplo, para identificar as condições de saúde das crianças, bem como para planejar a prestação dos serviços. Ao final, o diagnóstico situacional, ferramenta valiosa para produzir elementos indispensáveis ao entendimento da realidade de saúde com vistas a definir prioridade, é compartilhado em sala de aula (Figura 1).

Todas as atividades citadas que são desenvolvidas no espaço da gestão municipal ou regional configuram um ambiente de aprendizagem que se tornou tradição em algumas escolas médicas $^{7,18}$. Nesse ambiente, os alunos vivenciam as etapas do planejamento, regulação e programação, de forma a "descobrir" o universo que articula múltiplos atores e setores ${ }^{16}$. As entrevistas com os agentes para verificar pontos de estrangulamento, a 
Figura 1. Esquema do planejamento das ações dos alunos no serviço.

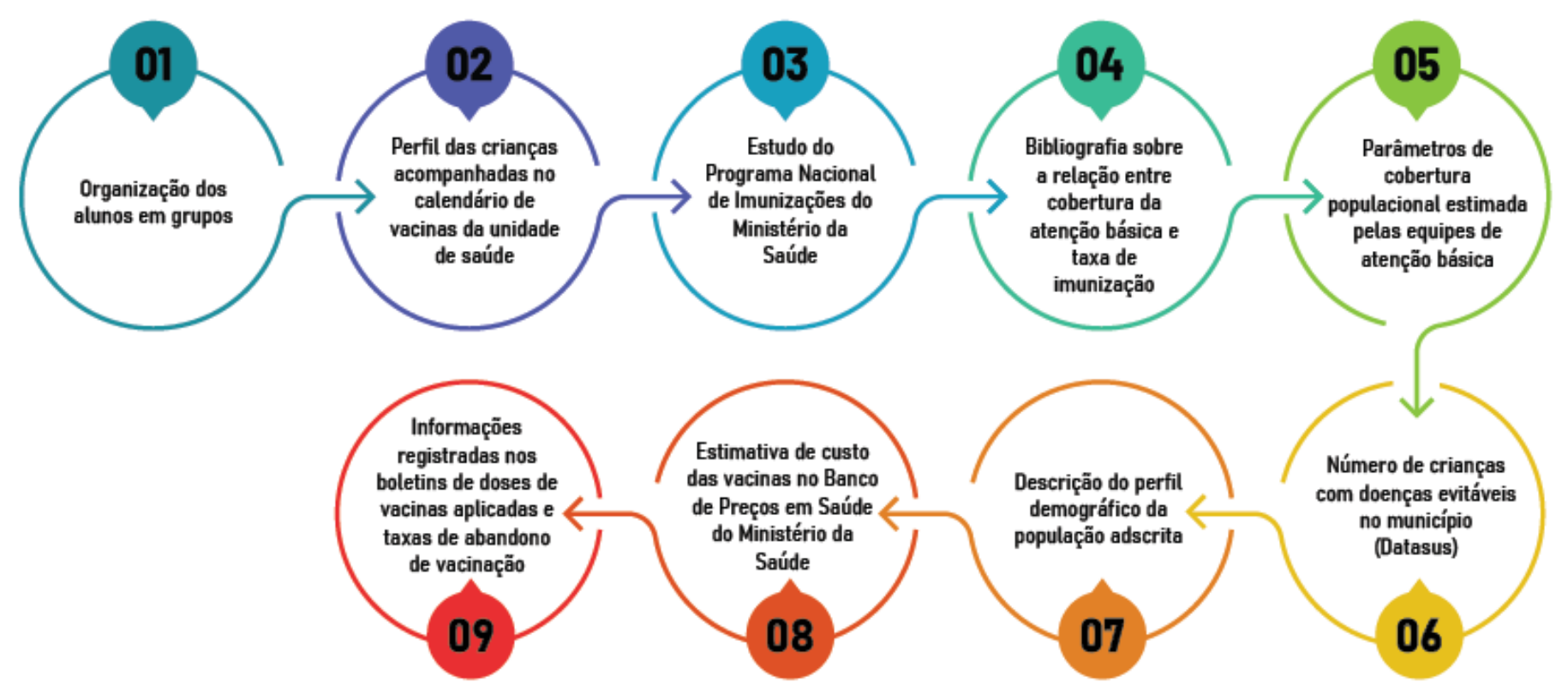

busca de informações nas bases de dados oficiais, a síntese de dados referentes ao perfil da população ou da infraestrutura disponível/necessária, entre outros procedimentos, são etapas que propiciarão a oportunidade de o aluno compreender a complexidade da organização dos sistemas de saúde ${ }^{18}$.

O contato direto com os atores desse serviço ensina que a resolução dos problemas depende da disponibilidade de recursos ${ }^{23}$. Ou seja, aprender no serviço de saúde é uma oportunidade para perceber a realidade e os diferentes graus de relevância consagrados pelas instituições aos problemas de saúde da população ${ }^{24}$. Participar dos grupos operativos ou das reuniões com os representantes da comunidade é uma aprendizagem sobre a produção social da saúde-doença, na medida em que problemas, conflitos e saídas são objeto da comunicação entre os agentes implicados ${ }^{25}$.

Em síntese, a elaboração de projeto ou a etapa do diagnóstico situacional é um potente objeto de aprendizagem da Saúde Coletiva, de maneira a justificar sua ampla disseminação nas matrizes curriculares das escolas médicas.

\section{Usar a sala de aula física de outro jeito}

O encontro na sala de aula física permite o cruzamento de várias modalidades de aprendizagem. Vimos, na seção anterior, a experiência de ensino baseado em projeto situacional. $\mathrm{Na}$ sequência, as técnicas da sala de aula invertida e da instrução por colegas ilustrarão as possibilidades para inovar a sala de aula tradicional.

\section{A sala de aula invertida}

A sala de aula invertida é um modelo pedagógico radical viabilizado pelos avanços da tecnologia instrucional. Para desenvolver um tema previsto na matriz curricular, o professor grava uma exposição dos aspectos de interesse e disponibiliza essa videoaula para o aluno acessar e assistir no momento que ele considerar adequado ${ }^{26}$.

Para os alunos da disciplina Saúde do Trabalhador, as videoaulas estão disponibilizadas na plataforma virtual de aprendizagem, na qual um roteiro orienta a atividade e indica os aspectos a serem focados na leitura principal. Como parte da atividade, um problema é apresentado no formato de relato de caso publicado em uma revista ou vivenciado pelo professor (ou pelos próprios alunos) no ambulatório. A situação ou o problema é o elemento motivador do estudo do caso, haja vista a sua potencialidade tanto de integrar conhecimentos e saberes quanto de produzir significados. Ocorrido ou fictício, o caso apresenta atributos do tema fundamental que se quer conhecer, sendo relacionado aos objetivos do ensino. Esses microtextos - videoaula, roteiro, leitura principal, estudo de caso - serão tratados na sequência, em momento e lugar escolhidos pelo aluno. A Figura 2 apresenta elementos da organização do processo ensino-aprendizagem descrito.

$O$ roteiro no formato de perguntas ou de assertivas que problematizam uma situação é útil para direcionar o estudo dos materiais ${ }^{27}$, como explicações sobre os diferenciais de exposição aos riscos ocupacionais. Uma vez concluída essa etapa, na sala de aula (invertida, portanto), a atividade vai requerer a interligação desses materiais, de maneira a constituir o objeto de aprendizagem. Por exemplo, a videoaula focalizou conhecimentos necessários para investigar a origem ocupacional das queixas de saúde, bem como as suas relações com o ambiente de trabalho. 
Figura 2. Planejamento das etapas da atividade na modalidade sala de aula invertida combinada com instrução por colegas.

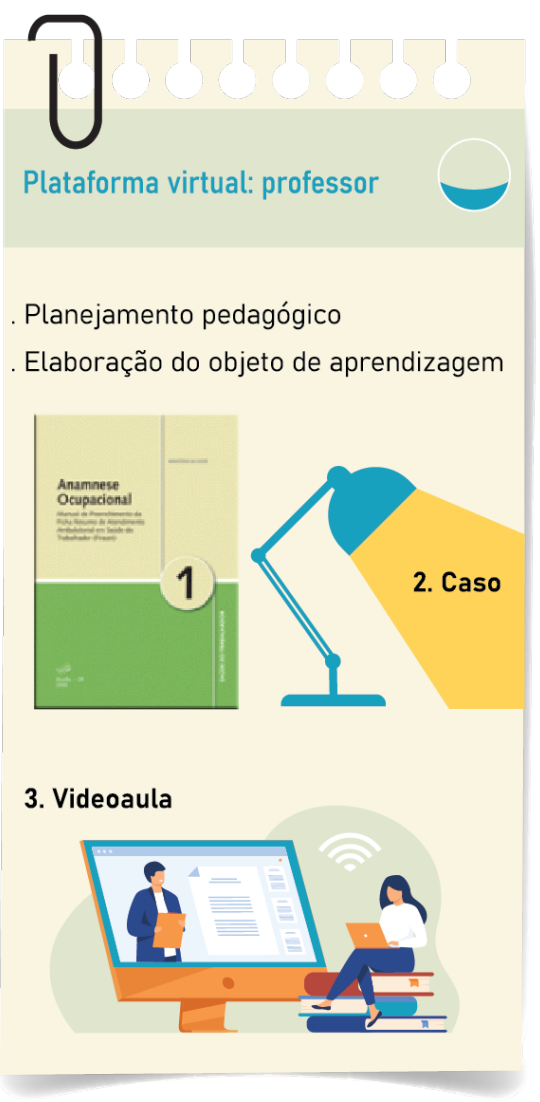

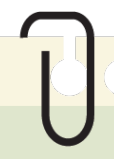

Estudo antes da aula: aluno

Depois de estudar o caso, responder às questões de acordo com leitura principal

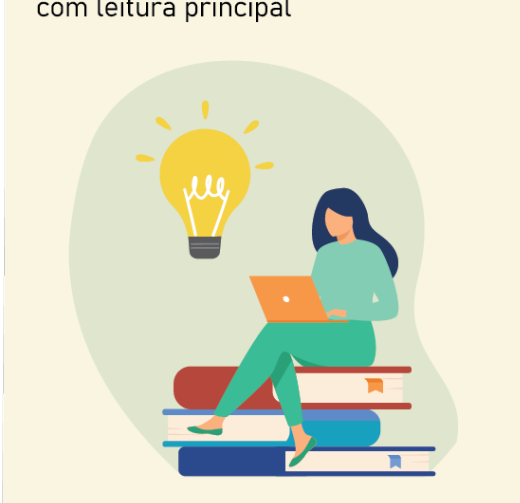

- Problema

excluir ou confirmar sua hipótese de origem ocupacional dos sintomas disfônicos relatados.

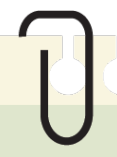

Sala de aula: alunos e professor

Instrução entre pares

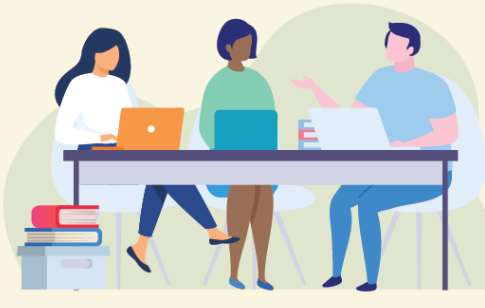

Devolutiva

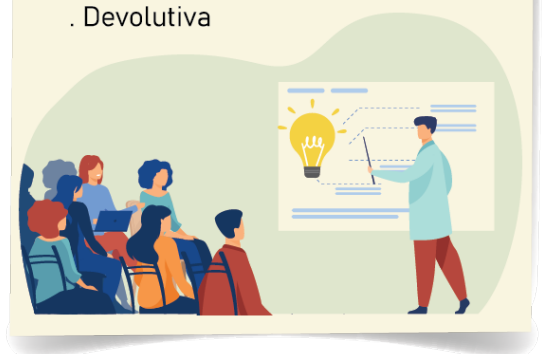

O protocolo de anamnese ocupacional do Ministério da Saúde foi a leitura principal, de maneira a oferecer um leque de dimensões do emprego e do trabalho relacionadas à produção social da doença. No roteiro, as perguntas foram relacionadas ao caso, cujas respostas dependeram da leitura do protocolo (p. 12) ${ }^{28}$.

O conjunto do material didático é estruturado e detalhadamente planejado, tendo em vista o respeito à cultura da geração atual que é mais afeita a percorrer espaços de forma circular, mais afinada com multitarefas e menos inclinada a dedicar atenção a uma única fonte de informações, conforme mencionado anteriormente. Em consideração a essa constatação, encara-se a sala de aula invertida como uma oportunidade para o professor estimular uma ampla gama de estilos de aprendizagem e desenvolver pedagogias que incentivam a resolução de problemas durante o tempo dedicado às aulas. Além disso, inverter a sala de aula abre portas para devolutivas no formato de diálogo aluno-professor-aluno e entre alunos ${ }^{3}$.

Em vez de longas exposições, o tempo na sala de aula foi destinado ao convívio necessário para trabalhar de forma colaborativa, de maneira a fazer emergir as reflexões e críticas elaboradas a jusante do encontro quando o aluno teve a seu dispor um roteiro e meios para explorar fontes cabíveis ${ }^{29}$. Nesse encontro, resultados das tarefas, reflexões sobre os problemas focados, entre outros, foram beneficiados da leitura precedente. Esses momentos se mostraram cruciais para reforçar ideais-chave em consonância ao plano de ensino, além de constituírem cenário favorável à reconstrução das ideias e dos conceitos, mantendo a preocupação de desenvolver maneiras pessoalmente significativas para o aluno.

\section{A instrução por colegas}

O método instrução por colegas consiste em apresentar, com antecedência ou em tempo real, assertivas ou perguntas aos alunos relacionadas aos conceitos do objeto da sessão. No passo seguinte, os alunos, em grupos ou individualmente, projetam os resultados por meio de dispositivos eletrônicos, quando for possível. Privilegia-se uma exposição dialogada com a finalidade de obter explicações de uns para os outros ${ }^{30}$. Em contraponto à aula tradicional, em que apenas os alunos mais participativos se envolvem a responder às questões abertas pelos professores, essa estratégia mostrou-se mais estimulante porque favoreceu a linguagem estruturada pelos próprios discentes ${ }^{31}$.

Dito de outro modo, a eficácia da instrução por colegas tem sido relacionada à transmissão por parte dos alunos de conhecimentos ou resolução de problemas para seus pares durante as discussões, de maneira a evitar a participação restrita a alguns discentes. A ação professor e 
alunos que se articulam para anunciar explicações cabíveis é uma forma de desenvolver a habilidade de colaborar para tratar de problemas complexos ${ }^{32}$. Essa situação pedagógica abre vias para o aluno anunciar conhecimentos na resolução de problemas, minimizando a ocorrência de dispersão do conteúdo. Contrastando respostas, estimulase mais o envolvimento dos presentes. Somente ao final, e quando necessário, a questão disparadora foi recuperada pelo professor com o objetivo de trabalhar o conceito fundamental que lhe diz respeito ${ }^{30}$.

A sala de aula invertida e a instrução por colegas apoiadas pelas tecnologias da informação e comunicação (TIC) conferiram um novo formato à sala de aula, de maneira a tornar a aprendizagem mais significativa. Em primeiro lugar, o uso das TIC facilitou a interação na sala de aula presencial ou virtual. A plataforma de aprendizagem, em segundo lugar, foi fundamental para o diálogo bidirecional professor-aluno, além da interação entre os colegas. Recursos em som e imagens viabilizaram, em terceiro lugar, a conexão dos materiais de forma a constituir um objeto de aprendizagem. $\mathrm{O}$ apoio da internet, em quarto lugar, é um benefício indispensável, uma vez que abre ao aluno a oportunidade de explorar livremente informações para desenvolver as atividades, de acordo com os caminhos que ele próprio escolher. Essa situação não dispensa os roteiros preparados para indicar fontes relevantes de consulta, como bibliotecas especializadas e instituições robustas na produção de sínteses relevantes de acordo com o estado da arte.

A devolutiva é uma ação avaliativa imprescindível quando as metodologias ativas de aprendizagem são empregadas $^{29}$. A organização dessa devolutiva foi crucial por pelo menos dois motivos. Primeiramente, foi uma maneira de sistematizar o conteúdo previsto no plano de ensino. Indicar aos alunos os pontos fortes da sua resenha ou sinalizar lapsos de interpretação, em segundo lugar, conferiu segurança a eles quanto à responsabilidade do professor na gestão do processo pedagógico. Por fim, estimular a explicitação ou a articulação do novo conteúdo ao repertório do aluno propiciou aprendizagens significativas, sendo mais eficiente do que considerar aquele momento como marco zero do processo.

\section{Constatando obstáculos e dificuldades}

Se, durante anos, o aluno tem convivido com a crença nos benefícios da aula expositiva, ou seja, centrada no professor-expositor, não foi surpreendente constatar hesitações por parte dos discentes nas atividades apoiadas pelas metodologias ativas. Esse tipo de manifestação requisita para o futuro próximo uma estratégia potente de avaliação, que seria favorecida pelas primeiras constatações. As críticas da turma relacionadas a deficiências de comunicação nas proposições de atividade foram encaradas como indicadores, servindo como orientações para rever a abordagem pedagógica. Como superar as nossas deficiências comunicativas para melhorar o diálogo na sala de aula?

Alguns alunos ressentiram-se do emprego de tempo necessário para interpretar as questões, mas sobretudo de ter de buscar eles próprios pelas fontes de conhecimento. Nesse caso, o que dizer das habilidades geracionais em interligar vários pontos, tratar concomitantemente várias fontes de informação e adotar um comportamento do tipo multitarefa4?

Esses fatos e impressões indicam a necessidade de construir um ambiente mais cooperativo e com mais recursos institucionais para persistir no caminho rumo a uma comunidade de aprendizagem. Provocar ações e estimular abstrações exigiu transposição didática (Figura 3), nos termos de Mallmann ${ }^{33}$. A elaboração de materiais didáticos, por exemplo, demandou treinamento do professor com vistas a produzir multimídia para incorporar textos, imagens, animações, vídeos e sons. Seria possível arquitetar uma estrutura de apoio tecnológico permanente aos professores investidos nas metodologias ativas de aprendizagem?

A inovação requer avanços na performance docente para conseguir planejar e empregar técnicas, fazer a gestão do calendário, produzir materiais e combinar procedimentos, elaborar devolutivas e aperfeiçoar as suas habilidades comunicacionais. 
Figura 3. Transposição didática e performance docente.

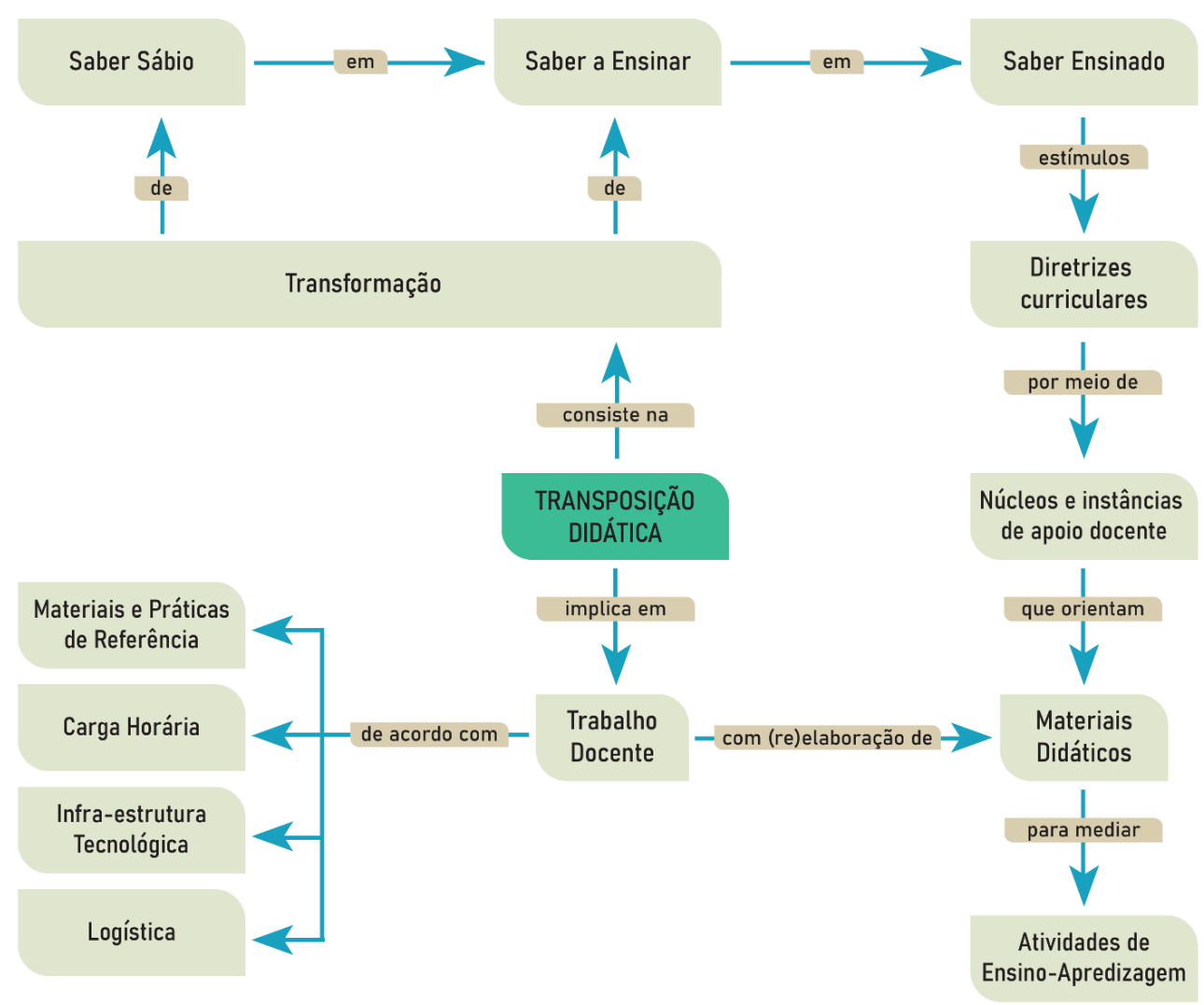

Fonte: Extraída e adaptada de Mallmann ${ }^{33}$.

\section{À GUISA DE CONCLUSÃO}

A elaboração de propostas pedagógicas baseadas nas metodologias ativas de aprendizagem considerou as características da geração do século XXI. Mas, se conexão e rede são valores para a geração atual que aprendeu a ter acesso à informação por seus próprios meios, viram-se tensões entre a passividade característica das situações tradicionais da sala de aula e a chamada para o envolvimento dos alunos em aulas dialogadas.

Amplamente reconhecidos os esforços institucionais, sem os quais não teria sido possível a experiência de que é objeto o presente artigo, a comunidade de aprendizagem, dessa vez, de professores merece reforço para compartilhar as melhores práticas e dialogar sobre os seus fracassos.

Por fim, a experiência em promover mudanças em direção a um modelo centrado no aluno requer avaliação criteriosa do impacto de sua implantação sobre os objetivos das diretrizes curriculares. Consta, na agenda dos próximos semestres, a elaboração de ferramentas para avaliação minuciosa da experiência relatada.

\section{CONFLITO DE INTERESSES}

Declaro não haver conflito de interesses.

\section{FINANCIAMENTO}

Fapemig APQ-04271-17.

\section{REFERÊNCIAS}

1. Vigotski LS. A formação social da mente. São Paulo: Martins Fontes; 2007.

2. Martins $\mathrm{OB}$, Moser A. Conceito de mediação em Vygotsky, Leontiev e Wertsch. Rev Intersaberes. 2012;7(13):8-28.

3. McLaughlin JE, Roth MT, Glatt DM, Gharkholonarehe N, Davidson CA, Griffin LM, et al. The flipped classroom: a course redesign to foster learning and engagement in a health professions school. Acad Med. 2014;89(2):236-43.

4. Cavert MS. Why flip the medical resident classroom? A pilot study in Singapore. In: Walker Z, Tan D, Koh NK, editors. Flipped classrooms with diverse learners. Singapore: Springer; 2020 [acesso em $3 \mathrm{dez}$ 2020]. Disponível em: https://www.semanticscholar.org/paper/ Why-Flip-the-Medical-Resident-Classroom-A-Pilot-in-Cavert/ b9a939494ce59b8c7fa591fdae5355ce00659c66.

5. Doueihi M. Pour un humanisme numérique. Paris: Seuil; 2011.

6. Stuart J, Rutherford RJ. Medical student concentration during lectures. Lancet. 1978;(2):514-6.

7. Funghetto SS, Silveir SM, Silvino AM, Karnikowski MGO. Perfil profissional tendo o SUS como base das Diretrizes Curriculares da Área da Saúde no processo avaliativo. Saúde Redes. 2015;1(3):103-20.

8. Silva CBG, Scherer MDDA. A implementação da Política Nacional de Educação Permanente em Saúde na visão de atores que a constroem. Interface Comun Saúde Educ. 2020;24:e190840.

9. Silva ALFD, Ribeiro MA, Paiva GMD, Freitas CASL, Albuquerque IMAN. Saúde e educação pelo trabalho: reflexões acerca do PET-Saúde como proposta de formação para o Sistema Único de Saúde. Interface Comun Saúde Educ. 2015;(19):975-84. 
10. Colegiado de Graduação do Curso de Medicina. Diretrizes Curriculares do Curso de Graduação em Medicina da UFMG. Documento aprovado por unanimidade em Reunião do Plenário do Colegiado ocorrida em 01/10/2008 [acesso em 9 jun 2021]. Disponível em: http://ftp.medicina. ufmg.br/cegrad/arquivos/2013/med/Diretrizes_Curriculares_FM_ UFMG_2009.pdf.

11. Freitas CM, Freitas CASL, Parente JRF, Vasconcelos MIO, Lima GK, Mesquita KOD, et al. Uso de metodologias ativas de aprendizagem para a educação na saúde: análise da produção científica. Trab Educ Saúde. 2015;(13):117-30.

12. Garcia MBDO, Oliveira MMD, Plantier AP. Interatividade e mediação na prática de metodologia ativa: o uso da instrução por colegas e da tecnologia na educação médica. Rev Bras Educ Med. 2019;43(1):87-96.

13. Marin MJS, Lima EFG, Paviotti AB, Matsuyama DT, Silva LKDD, Gonzalez $C$, et al. Aspectos das fortalezas e fragilidades no uso das metodologias ativas de aprendizagem. Rev Bras Educ Med. 2010;34(1):13-20.

14. Ramnanan CJ, Pound LD. Advances in medical education and practice: student perceptions of the flipped classroom. Adv Med Educ Pract. 2017;(8):63-73.

15. Lima CA, Rodrigues BG, Araújo JGC, Cardoso NR, Rocha PT, Ribeiro CDAL, et al. Diagnóstico situacional na unidade de saúde: uma experiência na perspectiva de graduandos em enfermagem. Rev Gest Saúde (Brasília). 2014;5(3):1109-19.

16. Silva REBG, Ramalho $A D P Q$, de Souza CCO. Oficinas de planejamento em saúde na disciplina de Saúde Coletiva: relato de experiência. Rev Graduação USP. 2018;3(2):87-91.

17. Amthauer C, Wolfart JM, da Silva AMF, Lebens DE, de Souza MG. A construção do diagnóstico situacional de saúde de uma estratégia saúde da família de São Miguel do Oeste, Santa Catarina. Anuário Pesquisa e Extensão Unoesc São Miguel do Oeste. 2020;5:e24613-e24613.

18. Maia ICVL, Peixoto MDGB, Schwermann LP, da Silva DLL, Maia DCG. Construções coletivas na formação médica sobre planejamento em saúde. Temas em Educação e Saúde. 2020;16(2):720-34.

19. Diniz RS, Araújo TAD. Integração ensino-serviço: experiência potencializada pelo Programa de Educação pelo Trabalho para a Saúde - Eixo Educação Permanente. Interface Comun Saúde Educ. 2019;23:e180540.

20. Queiroz RS, Valente GSC. Diagnóstico situacional em unidade básica de saúde: contribuições para o campo da saúde coletiva. Rev Enferm Atual In Derme. 2019;88(26):1-6.
21. Fonsêca GS, Junqueira SR. Programa de Educação pelo Trabalho para a Saúde da Universidade de São Paulo (Campus Capital): o olhar dos tutores. Cien Saude Colet. 2014;(19):1151-62.

22. Pinto APC, Pinto JC. Diagnóstico situacional da Unidade Básica de Saúde São Pedro. Rev Psico Saberes. 2020;9(17):153-65.

23. Batista $\mathrm{KBC}$, Gonçalves OSJ. Formação dos profissionais de saúde para o SUS: significado e cuidado. Saúde Soc. 2011;(20):884-99.

24. Kleba $M E$, Krauser IM, Vendruscolo $C$. O planejamento estratégico situacional no ensino da gestão em saúde da família. Texto Contexto Enferm. 2011;20(1):184-93.

25. Silva CSSL, Koopmans FF, Daher DV. O diagnóstico situacional como ferramenta para o planejamento de ações na atenção primária a saúde. Rev Pró-UniverSUS. 2016;7(2):30-3.

26. Schneider El, Suhr IRF, Rolon VEK, Almeida CM. Sala de aula invertida em EAD: uma proposta de Blended Learning. Rev Intersaberes. 2013;8(16):68-81.

27. Carvalho MWS, Andrade WZN, Cansanção IF, Palácio MAV, Takenami IO. Aprendizagem baseada em problemas como método de ensino na formação médica. Rev Doc Ens Sup. 2020;10:1-21.

28. Assunção AA. Disciplina Saúde do Trabalhador. Caderno de estudos. Belo Horizonte: Faculdade de Medicina da UFMG; 2013 [acesso em 09/06/2021]. Disponível em: https://www.medicina.ufmg.br/nest/ caderno-de-estudos/.

29. Valente JA. Blended learning e as mudanças no ensino superior: a proposta da sala de aula invertida. Educ Rev. 2014;(4):79-97.

30. Lasry N., Mazur E, Watkins J. Peer instruction: from Harvard to the two-year college. Am J Physics. 2008;76(11):1066-9.

31. Garcia MBDO, Oliveira MMD, Plantier AP. Interatividade e mediação na prática de metodologia ativa: o uso da instrução por colegas e da tecnologia na educação médica. Rev Bras Educ Med. 2019;43(1):87-96.

32. Relling $A E$, Giuliodori MJ. Effect of peer instruction on the likelihood for choosing the correct response to a physiology question. Adv Physio Educ. 2015;39(3):167-71.

33. Mallmann EM. Mediação pedagógica em educação a distância: inovação na docência universitária no processo de elaboração de materiais didáticos. RIED Rev Iberoam Educ Dist. 2010;13(1):157-89. 